General Letters in Mathematics (GLM) I(1)(2016) 23-31
General Letters in Mathematics (GLM)
Science
Reflection

\title{
Integral Equations and their Relationship to Differential Equations with Initial Conditions
}

\author{
Mostefa Nadir ${ }^{1}$, Somia Guechi ${ }^{2}$ \\ 1,2 Department of Mathematics, University of Msila, 28000, Algeria \\ 1 mostefanadir@yahoo.fr, 2 guechi.s2711@gmail.com
}

\begin{abstract}
Integral and differential equations have a fundamental importance in the functional analysis and the practice problems, and many domains of scientific research. However, the resolution of differential equations with constant coefficients is easy, but the resolution of these equations with variable coefficients is practically difficult or impossible in more part of the cases. This work present a analytical method which it transform a differential equations with initial conditions to a Volterra equations of second kind, efficient methods for approximate numerical solution of these equations, the analysis of the existence of their solutions, and the convergence of the error.
\end{abstract}

\section{Indexing terms/Keywords}

Differential equation, Volterra integral equation, Euler method, finite differences method, trapezoidal method.

\section{SUBJECT CLASSIFICATION}

MSC[2010]: 45D05, 45E05, 45L05, 45L10 and 65R20.

\section{Introduction}

We Two main types of integral equations will appear in this paper: their names occur in the table below. Suppose that $f:[a, b] \rightarrow \mathrm{R}$ and $k:[a, b]^{2} \rightarrow \mathrm{R}$ are continuous functions, and that $\lambda, a, b$ are constants.

Volterra non-homogeneous of second kind

$$
y(t)=f(t)+\lambda \int_{a}^{t} k(t, x) y(x) d x
$$

Volterra homogeneous of second kind

$$
y(t)=\lambda \int_{a}^{t} k(t, x) y(x) d x
$$

where $t \in[a, b]$, and the function $k=k(t, x)$ is called the kernelof the integral equation. 
A differential equation is an equation in which one or more variables, one or more functions of these variables and also the derivatives of these functions with respect to these variables occur the order of a differential equation is equal to the order of the highest occurring derivative.

In this paper we focus on second-order differential equations. We write a generic second-order equation for an unknown state $y=y(t)$ in the form

$$
\ddot{y}+A(t) \dot{y}+B(t) y=f(t)
$$

where $A, B$ and $f$ are known functions .

Further, most differential equations cannot be solved by performing a sequence of integrations, involving only elementary functions: polynomials, rational functions, trigonometric functions, exponentials, logarithms, and $A, B$ are constants.

For we find the relationship between integral and differential equations, we will need the following lemma which will allow us to replace a double integral by a single one.

Lemma1.1 (Replacement Lemma [ 1]) Suppose that $f:[a, b] \rightarrow R$ is continuous. Then

$$
\int_{a}^{t} \int_{a}^{t^{\prime}} f(x) d x d t^{\prime}=\int_{a}^{t}(t-x) f(x) d x, t \in[a, b] .
$$

Proof (See for example [1]).

\section{Transformation a differential equations to integral equations of Volterra kind}

In this section, let $I=[0, t]$, with $t<\infty$. We consider for example the problem of initial conditions following

$$
\left\{\begin{array}{l}
\ddot{y}+a(t) \dot{y}+b(t) y=f(t) \\
y(0)=A, \dot{y}(0)=B
\end{array}, t \in I \text { and } A, B \in R .\right.
$$

If we take

$\varphi(t)=\ddot{y}(t)$,

Integration from 0 to $t(t \in I)$ gives

$$
\begin{aligned}
\int_{0}^{t} \varphi(x) d x & =\int_{0}^{t} \ddot{y}(x) d x \\
& =\dot{y}(t)-\dot{y}(0) \\
& =\dot{y}(t)-B,
\end{aligned}
$$

then

$$
\int_{0}^{t} \phi(x) d x=\dot{y}(t)-B
$$

we can to write $y$ in the form

$$
\dot{y}(t)=\int_{0}^{t} \varphi(x) d x+B
$$


By integration the equation (3), we get

$$
\int_{0}^{t} \int_{0}^{x} \varphi(z) d z d x=y(t)-y(0)-B t .
$$

After the replacement lemma (1), we get

$$
\int_{0}^{t}(t-x) \varphi(x) d x=y(t)-A-B t
$$

Then

$$
y(t)=\int_{0}^{t}(t-x) \varphi(x) d x+B t+A
$$

If we replace the values of $y, \dot{y}$, and $\ddot{y}$ in the first equation of the problem (2), we will get an equation of the form

$$
\varphi(t)+a(t)\left[\int_{0}^{t} \varphi(x) d x\right]+b(t)\left[\int_{0}^{t}(t-x) \varphi(x) d x\right]=f(t)-B b(t) t-B a(t)+A b(t)
$$

then

$$
\varphi(t)-\int_{0}^{t} k(t, x) \varphi(x) d x=F(t)
$$

where

$$
k(t, x)=-a(t)-(t-x) b(t), \text { and } F(t)=f(t)-B b(t) t-B a(t)-A b(t) .
$$

Thus we have a non-homogeneous Volterra integral equation of the second kind.

The previous example indicates a fundamental relationship between Volterra integral equations and ordinary linear differential equation of the second-order. Actually, the solution of any differential equation of the type

$$
\frac{d^{n} y}{d t^{n}}+a_{1}(t) \frac{d^{n-1} y}{d t^{n-1}}+\cdots+a_{n}(t) y=f(t), \quad a \leq t \leq b
$$

with continuous coefficients, together with the initial conditions

$$
y(a)=C_{0}, \dot{y}(a)=C_{1}, \ldots, y^{(n-1)}(a)=C_{n-1} .
$$

Can be reduced to the solution of certain Volterra integral equation of the second kind

$$
\varphi(t)-\lambda \int_{a}^{t} k(t, x) \varphi(x) d x=F(t), \quad a \leq t \leq b
$$

where

$$
k(t, x)=-\sum_{k=1}^{n} a_{k}(t) \frac{(t-x)^{k-1}}{(k-1) !},
$$

and

$$
F(t)=f(t)-C_{n-1} a_{1}(t)-\left[(t-a) C_{n-1}-C_{n-2}\right] a_{2}(t)-\cdots-\left[\frac{(t-a)^{n-1}}{(n-1) !} C_{n-1}+\cdots+(t-a) C_{1}+C_{0}\right] a_{n}(t) .
$$




\section{Existence and Uniqueness of a Solution}

\subsection{For integral equation}

Theorem 3.1 (See for example [2]) Let $f \in \mathrm{L}_{2}[0,1]$, and suppose that $k$ is continuous for $t, x \in[0,1]$, and therefore uniformly bounded, say $|k(t, x)| \leq M$. Then the equation

$$
\varphi(t)-\int_{0}^{t} k(t, x) \varphi(x) d x=f(t),
$$

has a unique solution $\varphi$, for all $\lambda$ and $f$ in $\mathrm{L}_{2}[0,1]$.

Proof (See for example [2]).

\subsection{For Differential equation}

Reduction $n^{\text {th }}$ order differential equation to a system of $n$ first-order differential equations: Every explicit $n^{\text {th }}$ order differential equation (4) by introducing the new variables

$$
y_{1}=\dot{y}, y_{2}=\ddot{y}, \ldots, y_{n-1}=y^{(n-1)}
$$

can be reduced to a system of $n$ first-order differential equations

$$
\frac{d y}{d t}=y_{1}, \frac{d y_{1}}{d t}=y_{2}, \ldots, \frac{d y_{n-1}}{d t}=f\left(t, y, y_{1}, \ldots, y_{n-1}\right)
$$

Where $f$ is known function.

The system of $n$ differential equations

$$
\frac{d y_{i}}{d t}=f_{i}\left(t, y, y_{1}, \ldots, y_{n-1}\right), i=1,2, \ldots n
$$

has a unique system of solutions

$$
y_{i}=y_{i}(t),(i=1,2, \ldots, n)
$$

which is defined in an interval $t_{0}-h \leq t \leq t_{0}+h$ and for $t=t_{0}=a$ takes the previously given initial values

$$
y_{i}(a)=y_{i}^{0},(i=1,2, \ldots, n)
$$

if the functions $f_{i}\left(t, y, y_{1}, \ldots, y_{n-1}\right)$ are continuous with respect to all variables and satisfy the Lipschitz condition.

We ask that authors follow some simple guidelines. In essence, we ask you to make your paper look exactly like this document. The easiest way to do this is simply to download the template, and replace the content with your own material. Please use a 11-point Times New Roman font, , The goal is to have a 11-point text, as you see here.

\section{Numerical Solution of Linear Volterra Integral Equation of the Second kind}

The method of Trapezoidal is a method for constructing an approximate solution of an integral equation based on the replacement of integrals by finite sums according to some formula. Such formulas are called Trapezoidal formulas and, in general, have the form

$$
\int_{a}^{b} f(t) d t \simeq \sum_{i=1}^{n} \frac{\left(t_{i+1}-t_{i}\right)}{2}\left(f\left(t_{i}\right)+f\left(t_{i+1}\right)\right) .
$$


where $t_{i}(i=1, \ldots, n+1)$ are the abscissas of the partition points of the integration interval $[a, b]$, or interpolation nodes.

Let us choose a constant integration step $h=\frac{b-a}{n+1}=t_{i+1}-t_{i}$ and consider the discrete set of points $t_{i}=a+h(i-1), i=1, \ldots, n+1$. For $t=t_{i}$, the equation

$$
\varphi(t)-\int_{0}^{t} k(t, x) \varphi(x) d x=f(t), \text { and } \varphi(a)=f(a)
$$

acquires the form

$$
\phi\left(t_{j}\right)=f\left(t_{j}\right)-\int_{a}^{t_{j}} k\left(t_{j}, x\right) \phi(x) d x, \phi\left(t_{1}\right)=f\left(t_{1}\right), \quad a \leq t_{j} \leq b .
$$

Applying the Trapezoidal formula (5)to the integral in (6), we arrive at the system of equations

$$
\varphi_{j+1}=\frac{1}{1+\frac{h}{2} k_{j+1, j+1}}\left[f_{j+1}-\left(\frac{h}{2} k_{j+1,1} \varphi_{1}+h \sum_{i=2}^{j} k_{j+1, j} \varphi_{i}\right)\right] \text {, and } \varphi_{1}=f_{1} .
$$

where $\varphi\left(t_{j}\right)=\varphi_{j}, f\left(t_{j}\right)=f_{j}$ and $k\left(t_{j}, t_{i}\right)=k_{j, i}$, and $\varphi_{j}$ are approximate values of the unknown function at the nodes $t_{j}$ (see, for example, [ 3 ] ).

Corollary 4.1 (See, for example [4]) Let $k$ be a continuous kernel with

$$
\max _{t \in[a, b]}^{b} \int_{a}^{b}|k(t, x)| d x<1
$$

Then for all $f \in C([a, b])$, the integral equation of second kind

$$
\varphi(t)-\int_{0}^{t} k(t, x) \varphi(x) d x=f(t), t \in[a, b]
$$

has a unique solution $\varphi \in C([a, b])$, and the successive approximation

$$
\varphi_{n+1}(t)=\int_{a}^{t} k(t, x) \varphi_{n}(x) d x+f(t), \quad n=0,1,2, \ldots
$$

uniformly converge to the exact solution $\varphi$ for all $\varphi_{0} \in C([a, b]$.

Proof (See, for example [4]).

\section{Numerical Solution of second-order differential equation with initial conditions}

There are many numerical methods for the solution of differential equations of the second-order that can be used to obtain approximate solutions of differential equations. Such approximations are necessary when no exact solution can be found. In our study we will use two famous and simple methods Euler, and finite differences.

If we apply the Euler formula or finite differences (F-D) method in the problem of initial conditions (2), we will arrive at a linear system of equations (see, for example, [ 3 ] ). 


\section{Numerical Examples}

In this section we present a few numerical examples and all these examples given were run within MATLAB R2010a. First,

Let $y$ satisfy the differential equation with the initial values

$$
\left\{\begin{array}{l}
\ddot{y}(t)+t \dot{y}(t)+\exp (-t) y(t)=(1-t+\exp (-t)) \exp (-t), \quad 0 \leq t \leq 1 . \\
y(0)=1, \quad \dot{y}(0)=-1 .
\end{array}\right.
$$

This corresponds to the following Volterra integral equation

$$
y(t)-\int_{0}^{t}[-t+(x-t) \exp (-t)] y(x) d x=t+\exp (-2 t), \quad 0 \leq t \leq 1
$$

For which the exact solution is $\quad y(t)=\exp (-t)$.

Table 1. Comparison the results, absolute error for the integral equation (8).

\begin{tabular}{|c|c|c|c|}
\hline $\mathbf{t}$ & $y$ (exact) & $y_{n}$ by Trapezoidal method & error \\
\hline $\mathbf{0 . 0}$ & $1.0000 \mathrm{e}+000$ & $1.0000 \mathrm{e}+000$ & $0.0000 e+000$ \\
\hline $\mathbf{0 . 1}$ & $9.0484 \mathrm{e}-001$ & $9.0468 \mathrm{e}-001$ & $1.5427 e-004$ \\
\hline $\mathbf{0 . 2}$ & $8.1873 \mathrm{e}-001$ & $8.1845 \mathrm{e}-001$ & $2.8307 e-004$ \\
\hline $\mathbf{0 . 3}$ & $7.4082 \mathrm{e}-001$ & $7.4043 \mathrm{e}-001$ & $3.8658 e-004$ \\
\hline $\mathbf{0 . 4}$ & $6.7032 \mathrm{e}-001$ & $6.6985 \mathrm{e}-001$ & $4.6559 e-004$ \\
\hline $\mathbf{0 . 5}$ & $6.0653 \mathrm{e}-001$ & $6.0601 \mathrm{e}-001$ & $5.2146 e-004$ \\
\hline $\mathbf{0 . 6}$ & $5.4881 \mathrm{e}-001$ & $5.4826 \mathrm{e}-001$ & $5.5604 e-004$ \\
\hline $\mathbf{0 . 7}$ & $4.9659 \mathrm{e}-001$ & $4.9601 \mathrm{e}-001$ & $5.7158 e-004$ \\
\hline $\mathbf{0 . 8}$ & $4.4933 \mathrm{e}-001$ & $4.4876 \mathrm{e}-001$ & $5.7060 e-004$ \\
\hline $\mathbf{0 . 9}$ & $4.0657 \mathrm{e}-001$ & $4.0601 \mathrm{e}-001$ & $5.5580 e-004$ \\
\hline $\mathbf{1 . 0}$ & $3.6788 \mathrm{e}-001$ & $3.6735 \mathrm{e}-001$ & $5.2990 e-004$ \\
\hline
\end{tabular}

Table 2. Comparison the results, absolute error for differential equation (7)

\begin{tabular}{|c|c|c|c|c|}
\hline $\mathbf{t}$ & $y_{n}$ by Euler & error & $y_{n}$ by F-D & error \\
\hline $\mathbf{0 . 0}$ & $1.0000 \mathrm{e}+000$ & $0.0000 e+000$ & $1.0000 \mathrm{e}+000$ & $0.0000 e+000$ \\
\hline $\mathbf{0 . 1}$ & $9.0000 \mathrm{e}-001$ & $4.8374 e-003$ & $9.0000 \mathrm{e}-001$ & $4.8374 e-003$ \\
\hline $\mathbf{0 . 2}$ & $8.1100 \mathrm{e}-001$ & $7.7308 e-003$ & $8.0910 \mathrm{e}-001$ & $9.6344 e-003$ \\
\hline $\mathbf{0 . 3}$ & $7.3188 \mathrm{e}-001$ & $8.9356 e-003$ & $7.2647 \mathrm{e}-001$ & $1.4344 e-002$ \\
\hline $\mathbf{0 . 4}$ & $6.6161 \mathrm{e}-001$ & $8.7116 e-003$ & $6.5140 \mathrm{e}-001$ & $1.8924 e-002$ \\
\hline $\mathbf{0 . 5}$ & $5.9922 \mathrm{e}-001$ & $7.3156 e-003$ & $5.8319 \mathrm{e}-001$ & $2.3336 e-002$ \\
\hline $\mathbf{0 . 6}$ & $5.4382 \mathrm{e}-001$ & $4.9957 e-003$ & $5.2126 \mathrm{e}-001$ & $2.7548 e-002$ \\
\hline $\mathbf{0 . 7}$ & $4.9460 \mathrm{e}-001$ & $1.9854 e-003$ & $4.6505 \mathrm{e}-001$ & $3.1533 e-002$ \\
\hline $\mathbf{0 . 8}$ & $4.5083 \mathrm{e}-001$ & $1.5005 e-003$ & $4.1406 \mathrm{e}-001$ & $3.5272 e-002$ \\
\hline $\mathbf{0 . 9}$ & $4.1184 \mathrm{e}-001$ & $5.2704 e-003$ & $3.6782 \mathrm{e}-001$ & $3.8752 e-002$ \\
\hline $\mathbf{1 . 0}$ & $3.7704 \mathrm{e}-001$ & $9.1574 e-003$ & $3.2591 \mathrm{e}-001$ & $4.1965 e-002$ \\
\hline
\end{tabular}


Let $y$ satisfy the differential equation with the initial values

$$
\left\{\begin{array}{l}
\ddot{y}(t)+\frac{t}{4} \dot{y}(t)-\cos (t) y(t)=-\cos (t)\left(1+\cos (t)+\frac{t}{4} \sin (t)\right)+\frac{t}{4}, \quad 0 \leq t \leq 1 . \\
y(0)=1, \quad \dot{y}(0)=0 .
\end{array}\right.
$$

This corresponds to the following Volterra integral equation

$$
y(t)-\int_{0}^{t}\left[-\frac{t}{4}+(x-t) \cos (t)\right] y(x) d x=\frac{t}{4} \sin (t)+\cos ^{2}(t), \quad 0 \leq t \leq 1 .
$$

For which the exact solution is $\quad y(t)=\cos (t)$.

Table 3. Comparison of results, absolute error for the integral equation (10)

\begin{tabular}{|c|c|c|c|}
\hline$t$ & $y$ (exact) & $y_{n}$ by Trapezoidal method & error \\
\hline $\mathbf{0 . 0}$ & $1.0000 \mathrm{e}+000$ & $1.0000 \mathrm{e}+000$ & $0.0000 e+000$ \\
\hline $\mathbf{0 . 1}$ & $9.9500 \mathrm{e}-001$ & $9.9501 \mathrm{e}-001$ & $6.2169 e-006$ \\
\hline $\mathbf{0 . 2}$ & $9.8007 \mathrm{e}-001$ & $9.8009 \mathrm{e}-001$ & $2.4536 e-005$ \\
\hline $\mathbf{0 . 3}$ & $9.5534 \mathrm{e}-001$ & $9.5539 \mathrm{e}-001$ & $5.3968 e-005$ \\
\hline $\mathbf{0 . 4}$ & $9.2106 \mathrm{e}-001$ & $9.2115 \mathrm{e}-001$ & $9.2886 e-005$ \\
\hline $\mathbf{0 . 5}$ & $8.7758 \mathrm{e}-001$ & $8.7772 \mathrm{e}-001$ & $1.3906 e-004$ \\
\hline $\mathbf{0 . 6}$ & $8.2534 \mathrm{e}-001$ & $8.2553 \mathrm{e}-001$ & $1.8969 e-004$ \\
\hline $\mathbf{0 . 7}$ & $7.6484 \mathrm{e}-001$ & $7.6508 \mathrm{e}-001$ & $2.4153 e-004$ \\
\hline $\mathbf{0 . 8}$ & $6.9671 \mathrm{e}-001$ & $6.9700 \mathrm{e}-001$ & $2.9092 e-004$ \\
\hline $\mathbf{0 . 9}$ & $6.2161 \mathrm{e}-001$ & $6.2194 \mathrm{e}-001$ & $3.3396 e-004$ \\
\hline $\mathbf{1 . 0}$ & $5.4030 \mathrm{e}-001$ & $5.4067 \mathrm{e}-001$ & $3.6665 e-004$ \\
\hline
\end{tabular}

Tab 4. Comparison des results, absolute error for differential equation (9)

\begin{tabular}{|c|c|c|c|c|}
\hline $\mathbf{t}$ & $y_{n}$ by Euler & error & $y_{n}$ by F-D & error \\
\hline $\mathbf{0 . 0}$ & $1.0000 \mathrm{e}+000$ & $0.0000 e+000$ & $1.0000 \mathrm{e}+000$ & $0.0000 e+000$ \\
\hline $\mathbf{0 . 1}$ & $1.0000 \mathrm{e}+000$ & $4.9958 e-003$ & $1.0000 \mathrm{e}+000$ & $4.9958 e-003$ \\
\hline $\mathbf{0 . 2}$ & $9.9000 \mathrm{e}-001$ & $9.9334 e-003$ & $9.9015 \mathrm{e}-001$ & $1.0083 e-002$ \\
\hline $\mathbf{0 . 3}$ & $9.7005 \mathrm{e}-001$ & $1.4714 e-002$ & $9.7079 \mathrm{e}-001$ & $1.5456 e-002$ \\
\hline $\mathbf{0 . 4}$ & $9.4040 \mathrm{e}-001$ & $1.9340 e-002$ & $9.4246 \mathrm{e}-001$ & $2.1404 e-002$ \\
\hline $\mathbf{0 . 5}$ & $9.0150 \mathrm{e}-001$ & $2.3916 e-002$ & $9.0588 \mathrm{e}-001$ & $2.8296 e-002$ \\
\hline $\mathbf{0 . 6}$ & $8.5398 \mathrm{e}-001$ & $2.8649 e-002$ & $8.6191 \mathrm{e}-001$ & $3.6578 e-002$ \\
\hline $\mathbf{0 . 7}$ & $7.9868 \mathrm{e}-001$ & $3.3839 e-002$ & $8.1160 \mathrm{e}-001$ & $4.6757 e-002$ \\
\hline $\mathbf{0 . 8}$ & $7.3658 \mathrm{e}-001$ & $3.9874 e-002$ & $7.5609 \mathrm{e}-001$ & $5.9385 e-002$ \\
\hline $\mathbf{0 . 9}$ & $6.6883 \mathrm{e}-001$ & $4.7220 e-002$ & $6.9666 \mathrm{e}-001$ & $7.5045 e-002$ \\
\hline $\mathbf{1 . 0}$ & $5.9671 \mathrm{e}-001$ & $5.6405 e-002$ & $6.3463 \mathrm{e}-001$ & $9.4325 e-002$ \\
\hline
\end{tabular}


Let $y$ satisfy the differential equation with the initial values

$$
\left\{\begin{array}{l}
\ddot{y}(t)-\frac{1}{t+2} \dot{y}(t)+\frac{t}{2} y(t)=\frac{t^{4}+2 t^{3}+2 t^{2}+4 t+8}{2 t+2}, \quad 0 \leq t \leq 1 . \\
y(0)=2, \quad \dot{y}(0)=0 .
\end{array}\right.
$$

This corresponds to the following Volterra integral equation

$$
y(t)-\int_{0}^{t}\left[\frac{1}{t+2}+\frac{t}{2}(x-t)\right] y(x) d x=\frac{1}{24}\left(t^{5}+12 t^{3}\right)-\frac{t^{3}+6 t}{3 t+6}+t^{2}+2, \quad 0 \leq t \leq 1 .
$$

For which the exact solution is $y(t)=t^{2}+2$.

Table 5. Comparison of results, absolute error for the integral equation (12).

\begin{tabular}{|c|c|c|c|}
\hline $\mathbf{t}$ & $y$ (exact) & $y_{n}$ by Trapezoidal method & error \\
\hline $\mathbf{0 . 0}$ & $2.0000 \mathrm{e}+000$ & $2.0000 \mathrm{e}+000$ & $0.0000 e+000$ \\
\hline $\mathbf{0 . 1}$ & $2.0100 \mathrm{e}+000$ & $2.0101 \mathrm{e}+000$ & $8.1728 e-005$ \\
\hline $\mathbf{0 . 2}$ & $2.0400 \mathrm{e}+000$ & $2.0402 \mathrm{e}+000$ & $1.6217 e-004$ \\
\hline $\mathbf{0 . 3}$ & $2.0900 \mathrm{e}+000$ & $2.0902 \mathrm{e}+000$ & $2.4406 e-004$ \\
\hline $\mathbf{0 . 4}$ & $2.1600 \mathrm{e}+000$ & $2.1603 \mathrm{e}+000$ & $3.3002 e-004$ \\
\hline $\mathbf{0 . 5}$ & $2.2500 \mathrm{e}+000$ & $2.2504 \mathrm{e}+000$ & $4.2251 e-004$ \\
\hline $\mathbf{0 . 6}$ & $2.3600 \mathrm{e}+000$ & $2.3605 \mathrm{e}+000$ & $5.2378 e-004$ \\
\hline $\mathbf{0 . 7}$ & $2.4900 \mathrm{e}+000$ & $2.4906 \mathrm{e}+000$ & $6.3591 e-004$ \\
\hline $\mathbf{0 . 8}$ & $2.6400 \mathrm{e}+000$ & $2.6408 \mathrm{e}+000$ & $7.6068 e-004$ \\
\hline $\mathbf{0 . 9}$ & $2.8100 \mathrm{e}+000$ & $2.8109 \mathrm{e}+000$ & $8.9961 e-004$ \\
\hline $\mathbf{1 . 0}$ & $3.0000 \mathrm{e}+000$ & $3.0011 \mathrm{e}+000$ & $1.0538 e-003$ \\
\hline
\end{tabular}

Table 6. Comparison des results, absolute error for differential equation (11).

\begin{tabular}{|c|c|c|c|c|}
\hline $\mathbf{t}$ & $\mathbf{y}_{n}$ byEuler & error & $\mathbf{y}_{n}$ by F-D & error \\
\hline $\mathbf{0 . 0}$ & $2.0000 \mathrm{e}+000$ & $0.0000 e+000$ & $2.0000 \mathrm{e}+000$ & $0.0000 e+000$ \\
\hline $\mathbf{0 . 1}$ & $2.0000 \mathrm{e}+000$ & $1.0000 e-002$ & $2.0000 \mathrm{e}+000$ & $1.0000 e-002$ \\
\hline $\mathbf{0 . 2}$ & $2.0200 \mathrm{e}+000$ & $2.0000 e-002$ & $2.0200 \mathrm{e}+000$ & $1.9995 e-002$ \\
\hline $\mathbf{0 . 3}$ & $2.0600 \mathrm{e}+000$ & $3.0005 e-002$ & $2.0600 \mathrm{e}+000$ & $2.9968 e-002$ \\
\hline $\mathbf{0 . 4}$ & $2.1200 \mathrm{e}+000$ & $4.0030 e-002$ & $2.1201 \mathrm{e}+000$ & $3.9894 e-002$ \\
\hline $\mathbf{0 . 5}$ & $2.1999 \mathrm{e}+000$ & $5.0101 e-002$ & $2.2003 \mathrm{e}+000$ & $4.9733 e-002$ \\
\hline $\mathbf{0 . 6}$ & $2.2997 \mathrm{e}+000$ & $6.0256 e-002$ & $2.3006 \mathrm{e}+000$ & $5.9435 e-002$ \\
\hline $\mathbf{0 . 7}$ & $2.4195 \mathrm{e}+000$ & $7.0540 e-002$ & $2.4211 \mathrm{e}+000$ & $6.8940 e-002$ \\
\hline $\mathbf{0 . 8}$ & $2.5590 \mathrm{e}+000$ & $8.1014 e-002$ & $2.5618 \mathrm{e}+000$ & $7.8176 e-002$ \\
\hline $\mathbf{0 . 9}$ & $2.7183 \mathrm{e}+000$ & $9.1747 e-002$ & $2.7229 \mathrm{e}+000$ & $8.7059 e-002$ \\
\hline $\mathbf{1 . 0}$ & $2.8972 \mathrm{e}+000$ & $1.0282 e-001$ & $2.9045 \mathrm{e}+000$ & $9.5497 e-002$ \\
\hline
\end{tabular}




\section{Conclusion}

we can conclude all differential equation with initial conditions corresponds to Volterra integral equation of the second kind, and the numerical solution of integral equation (for example by Trapezoidal method) is the better than the numerical solution of correspondent differential equation (for example by Euler or finite differences method).

\section{References}

[1] P. Collins, Differential and integral equation, Oxford, university of Press, New York, 2006.

[2] H. Hochstadt.Integral equations, Polytechnic instite of Brooklyn, New York, 1973.

[3] S. Guechi.Relation des équations integrales et differentielles, Thesis of Master university of Msila, Algeria, 2012.

[4] M. Nadir. Cours sur les équations intégrales. University of Msila, Algeria, 2008. 